\title{
Letter to the Editor concerning "Risk factors for surgical site infection and urinary tract infection after spine surgery" by Tominaga, H, Setoguchi, T, Ishidou, Y, Nagano, S, Yamamoto, T, Komiya S (2016) Eur Spine J; doi:10.1007/s00586-016-4674-2
}

\author{
Hua Zhong ${ }^{1} \cdot$ Zhihong Zhou $^{1} \cdot$ Jianchun Liu $^{1} \cdot$ Siyang Huang $^{2} \cdot$ Jia Huang $^{3}$
}

Received: 16 August 2016/ Accepted: 8 September 2016/Published online: 21 September 2016

(c) Springer-Verlag Berlin Heidelberg 2016

Dear Editor,

It was with great interest that I read the study entitled 'Risk factors for surgical site infection and urinary tract infection after spine surgery.' published online in June 2016 in European Spine Journal [1]. In this paper, the authors report that risk factors for post-operative surgical site infection and urinary tract infection were operative time and ASA classification 3. It is an interesting study. Nevertheless, we have some queries which we would like to communicate with the authors.

As is a retrospective observational study, only association and not causation could be inferred from the results of the above cohort study. It was not possible to measure and then control for the confounding via statistical analysis in the retrospective study. In this study, the authors did not exclude risk factors of UTIs preoperative such as urinary tract abnormalities, blockages in the urinary tract, a recent urinary procedure and asymptomatic UTIs, which may affect the incidence of UTIs secondary to the spine surgery. Moreover, before the surgery, the patients did not undergo urinalysis. The authors cannot differentiate that spine surgery was the primary or secondary risk factor of postoperative UTIs.

\section{Compliance with ethical standards}

Conflict of interest The authors declare that they have no conflict of interest.

\section{Reference}

1. Tominaga H, Setoguchi T, Ishidou Y, Nagano S, Yamamoto T, Komiya S (2016) Risk factors for surgical site infection and urinary tract infection after spine surgery. Eur Spine J. doi:10. 1007/s00586-016-4674-2
Jia Huang

huangjia10@csu.edu.cn

1 Department of Orthopedics Surgery, Central Hospital of Yi

Yang, Yiyang, Hunan Province, China

2 XiangXing College of Hunan University of Chinese Medicine, Changsha, Hunan Province, China

3 Department of Intensive Care Unit, The Second Xiangya Hospital, Central South University, Changsha 410011, China 